\title{
Continuum Description of the Cytoskeleton: Ring Formation in the Cell Cortex
}

\author{
Alexander Zumdieck ${ }^{1} *$ Marco Cosentino Lagomarsino ${ }^{2, *}$ U Catalin Tanase ${ }^{2, *}$ 团 \\ Karsten Kruse ${ }^{1}$, Bela Mulder ${ }^{2}$, Marileen Dogterom ${ }^{2}$, and Frank Jülicher ${ }^{1}$ \\ ${ }^{1}$ Max-Planck-Institut für Physik komplexer Systeme, Dresden, Germany and \\ ${ }^{2}$ FOM Institute for Atomic and Molecular Physics (AMOLF), Amsterdam, The Netherlands
}

\begin{abstract}
Motivated by the formation of ring-like filament structures in the cortex of plant and animal cells, we study the dynamics of a two-dimensional layer of cytoskeletal filaments and motor proteins near a surface by a general continuum theory. As a result of active processes, dynamic patterns of filament orientation and density emerge via instabilities. We show that self-organization phenomena can lead to the formation of stationary and oscillating rings. We present state diagrams which reveal a rich scenario of asymptotic behaviors and discuss the role of boundary conditions.
\end{abstract}

PACS numbers: 87.17.-d, 87.16.Ka, 05.65.+b

The cytoskeleton, an organized network of filamentous proteins, is an essential component of all eukaryotic cells. It plays a major role in morphogenesis, transport, motility and cell division [1]. The key components of the cytoskeleton are actin filaments and microtubules which are long elastic protein filaments that reach lengths of several $\mu \mathrm{m}$. They have chemically and dynamically distinct ends and hence are structurally polar. Motor proteins consume chemical fuel to generate directed motion on these filaments and hence can induce stresses in the cytoskeletal network.

An important cytoskeletal structure in various cell types are cortical rings formed by bundles of filaments which wrap around the cell. Such rings form within the cell cortex, a thin layer of filament network located close to the cell membrane. In animal cells a contractile ring containing actin filaments, cleaves the dividing cell by generating a constriction [1. In higher plant cells, microtubules form cortical arrays (CA) between consecutive cell divisions 2], with a preferred orientation in the azimuthal direction, see Fig. 1(a). Shortly before entering division, the $\mathrm{CA}$ is dynamically reorganized and the preprophase band (PPB) is formed [2]. It consists of a ring-like bundle of microtubules that determines the location and orientation of the future division plane of the cell [3] , see Fig. प(b). In addition to single rings, double rings have been observed in certain cells [4].

It has been experimentally observed in vitro that mixtures of microtubules and motor proteins can selforganize into a number of patterns, such as bundles, asters and vortices 5, 6]. Similar patterns where found in molecular dynamics simulations 6, 7]. Theoretical descriptions of cytoskeletal dynamics are either based on microscopic models for the motor-filament interactions [8, 9, 10, 11] or consider the behavior on large length and time scales in terms of a continuum theory 12, 13, 14, 15, 16, 17. In contrast to microscopic descriptions, which rely on specific and potentially incomplete microscopic pictures of the nonequilibrium driving forces, a phenomenological description captures generic conditions for the formation of patterns through insta-

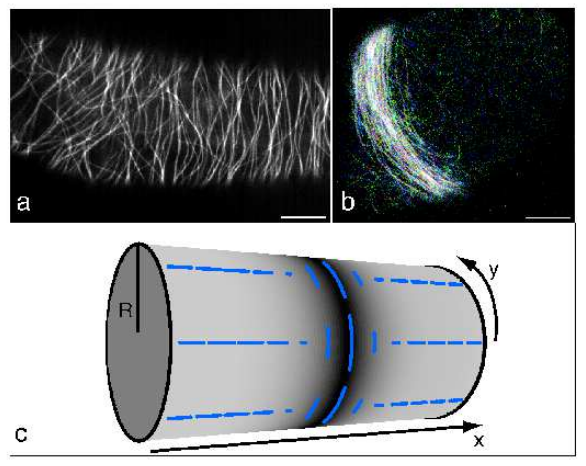

FIG. 1: (color online) Density and orientation of cortical filaments. (a,b) Fluorescence microscopy images of microtubule patterns in Tobacco BY2 suspension cells. (a) Between cell divisions microtubules form a cortical array and orient preferentially perpendicular to the cell's long axis. (b) Shortly before cell division microtubules condense into a ring-like structure, the Pre-Prophase Band (PPB). (c) Representation of a stationary solution to the dynamic equations (1)-(5) resembling a PPB. The density of filaments is represented by the grey level on the cylinder. The blue bars indicate the nematic order of filaments by their orientation and length. (a,b) courtesy of Jan Vos, Wageningen University. Scale bars are $10 \mu \mathrm{m}$.

bilities. In the present work we develop a general coarse grained description of the two-diemensional cell cortex based on symmetry arguments and extend previous work to include nematic order.

We focus on the dynamical behaviour of a cortical layer of filaments at scales large compared to the filament lengths and the layer thickness, where a coarse grained, two-dimensional description is suitable. We assume that the state of the system is essentially specified by the scalar mass density $c(\boldsymbol{r}, t)$, the polarization $\boldsymbol{p}(\boldsymbol{r}, t)$, a vector along the average direction of the filaments, and $\boldsymbol{q}(\boldsymbol{r}, t)$, a second rank traceless symmetric tensor that measures orientational order. These fields represent slow hydrodynamic modes as they are linked either to a conservation law (the density $c$ ) or to a spontaneously broken symmetry (the fields $\boldsymbol{p}$ and $\boldsymbol{q}$ ). Other degrees of freedom 
in the system are assumed to relax more rapidly and are thus ignored [16].

The choice of the appropriate dynamic equations for these fields is governed by the following principles (i) all dynamics is overdamped as its origin lies in the motion of colloidal sized particles in a viscous background fluid, (ii) all mass transport is due to stresses generated in the system, and (iii) all driving terms are allowed that respect the rotational invariance of space. This is consistent with the fact that they are caused by local interactions between the microscopic components. For the mass density we have the continuity equation

$$
\partial_{t} c+\nabla \cdot \boldsymbol{j}=s
$$

where $\boldsymbol{j}$ is the filament current and $s$ represents source and sink terms describing the polymerization and depolymerization of filaments. The filament current is a consequence of inhomogeneities in the mechanical stress in the filament system. For a layer of cortical filaments close to the cell surface moving against an immobile background fluid and assuming local isotropic friction, we thus write

$$
\boldsymbol{j}=\eta^{-1} \nabla \cdot \boldsymbol{\sigma}
$$

where $\boldsymbol{\sigma}$ is the stress tensor and $\eta$ an effective friction coefficient. The dynamic equations for the non-conserved fields $\boldsymbol{p}$ and $\boldsymbol{q}$ can be expressed as $\partial_{t} \boldsymbol{p}=\boldsymbol{\psi}$, and $\partial_{t} \boldsymbol{q}=\boldsymbol{\omega}$, where $\boldsymbol{\psi}$ is a vector and $\boldsymbol{\omega}$ is a symmetric traceless tensor.

These equations are closed by assuming that the evolution of the system is completely determined by the present state of the system. This implies that the stress tensor $\boldsymbol{\sigma}$ and the "velocities" of the polarization and the nematic order parameter $\boldsymbol{\psi}$ and $\boldsymbol{\omega}$ can be expressed in terms of the dynamic fields $c, \boldsymbol{p}$ and $\boldsymbol{q}$. We then expand these driving forces in terms of perturbations of the dynamic fields and their spatial derivatives around a reference state that we assume to be homogenous.

We describe the cell cortex as a two-dimensional active gel, embedded in three dimensional space along the cell surface. Motivated by the morphology of plant cells, we wrap the $2 \mathrm{~d}$ gel on a cylinder. Experimental evidence indicates that in the CA filaments orient in a non-polar way 18. It has been suggested that this holds for the PPB as well [19, 20]. Therefore, we neglect the polarization $\mathbf{p}$ and only keep the nematic order $\mathbf{q}$. In situations where the total mass of polymerized microtubules is conserved (such as the transition from CA to PPB [21]), the source term $s$ can be neglected. For simplicity, we focus on states of rotational symmetry around the cylinder axis and we neglect effects of the surface curvature.

The cylinder axis is the $x$-axis of our coordinate frame, the second dimension described by the $y$-axis is wrapped around the cylinder with radius $R$, see Fig. 11 (c). The dynamics of configurations which are rotationally invariant, obey a set of equations projected on the cylinder axis, which can be derived by symmetry arguments [12, 15]:

$$
\begin{aligned}
\eta^{-1} \sigma_{x x}= & A_{1} c+A_{2} c^{2}+A_{3} c \partial_{x}^{2} c+A_{4}\left(\partial_{x} c\right)^{2}+A_{5} q_{x}(3) \\
\omega_{x x}= & -B_{1} q_{x x}+B_{2} \partial_{x}^{2} q_{x x}+B_{3} \partial_{x}^{4} q_{x x}+B_{4} \partial_{x}^{2} c \quad(4) \\
& +B_{5}\left(q_{x x}^{2}+q_{x y}^{2}\right) q_{x x} \\
\omega_{x y}= & -B_{1} q_{x y}+B_{2} \partial_{x}^{2} q_{x y}+B_{3} \partial_{x}^{4} q_{x y} \\
& +B_{5}\left(q_{x x}^{2}+q_{x y}^{2}\right) q_{x y}
\end{aligned}
$$

where we have neglected higher order terms. It has been shown that the terms in Eq. (3)-(5) arise from coarsegrained microscopic models and can be related to filament sliding induced by motor proteins in an immobile viscous background 8, 11, 15, 27]. An example is the contractile tension given by the term $A_{2} c^{2}$ which has been discussed in [15].

Dimensional analysis reveals that these equations contain six independent parameters. The values of only three of these turn out to influence the dynamics qualitatively; they can be identified with $A_{2}, B_{2}$, and $F=-A_{5} B_{4}$. The other parameters describe nonlinear terms which are required to stabilize the dynamics. Stability requires $A_{3}>0, A_{4}<0, B_{3}<0$, and $B_{5}<0$. The specific values of these parameters do not qualitatively influence the asymptotic dynamics.

In order to get some general insight into this problem, we first consider the somewhat artificial case of periodic boundary conditions in $x$-direction with period $L$. In this case, the homogenous isotropic state, i.e., $c(x)=c_{0}$, $q_{x x}(x)=q_{x y}(x)=0$ for all $x$, is a stationary solution of the dynamic equations (3)-(5). We first perform a linear stability analysis of this state. The linearized equation for the perturbation $\delta q_{x y}$ decouples from the corresponding equations for the perturbations $\delta c$ and $\delta q_{x x}$. The field $q_{x y}$ becomes unstable for $B_{1}<0$. This instability corresponds to the isotropic-nematic transition. Here, we focus on the instabilities of the isotropic homogenous state with $B_{1}>0$.

The linearized dynamics for $\delta c=\sum_{n} c_{k} \mathrm{e}^{2 \pi i n x / L}$ and $\delta q_{x x}=\sum_{n} q_{x x, k} \mathrm{e}^{2 \pi i n x / L}$ is given by

$$
\begin{aligned}
& \frac{d}{d t}\left(\begin{array}{c}
c_{k} \\
q_{x x, k}
\end{array}\right)= \\
& \quad\left(\begin{array}{cc}
-D k^{2}-A_{3} c_{0} k^{4} & +A_{5} k^{2} \\
-B_{4} k^{2} / 2 & -B_{1}-B_{2} k^{2}+B_{3} k^{4}
\end{array}\right)\left(\begin{array}{c}
c_{k} \\
q_{x x, k}
\end{array}\right),
\end{aligned}
$$

where $D=-\left(A_{1}+2 c_{0} A_{2}\right)$ is an effective diffusion constant. Figure 2 represents the region of stability of the homogenous isotropic state as a dark grey area for sufficiently large values of the parameters $\tilde{D}$ and $\tilde{B}_{2}$. The parameter $-\tilde{D}$ is related to the contractile tension in the system. For sufficiently small $\tilde{D}$, the system tends to undergo density instabilities which lead to ring solutions. The parameter $\tilde{B}_{2}$ characterizes a length scale related to the orientational order. For sufficiently small $\tilde{B}_{2}$ orientational instabilities occur. The corresponding unstable 


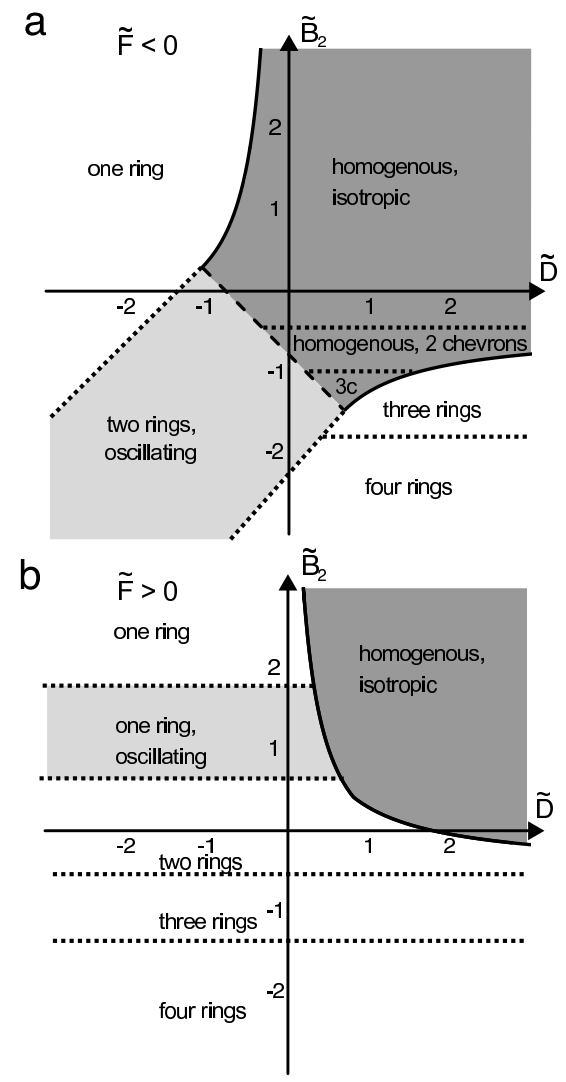

FIG. 2: Schematic state diagrams of an active gel layer in cylindrical geometry with periodic boundary conditions in the $x$-direction, are shown for two values of $\tilde{F}$ as a function of two dimensionless parameters $\tilde{B}_{2}$ and $\tilde{D}$. Regions of linear stability of homogeneous filament distributions are shaded in dark grey. Outside these regions, the topology of the state diagram as determined numerically is indicated by dotted lines. Asymptotic states include chevron patterns and single or multiple rings that are either stationary or do oscillate. Regions of oscillating solutions are shaded in light grey. The long dashed line indicates a Hopf bifurcation determined by linear stability analysis. Examples for stationary chevron and ring patterns are displayed in Fig. 3] Parameters are defined as $\tilde{D}=-\left(\tilde{A}_{1}+2 \tilde{c}_{0} \tilde{A}_{2}\right), \tilde{B}_{2}=B_{2} /\left(B_{1} L^{2}\right), \tilde{F}=F /\left(B_{1}^{2} L^{4}\right)$ and $\tilde{A}_{2}=A_{2} /\left(B_{1} L^{4}\right)$. Parameters values are $\tilde{c}_{0}=c_{0} L=0.5$, $L\left(-2 B_{1} / A_{1}\right)^{1 / 2}=5, \tilde{A}_{1}=A_{1} /\left(B_{1} L^{2}\right)=-2, A_{3} /\left(B_{1} L^{6}\right)=$ $0.1, A_{4} /\left(B_{1} L^{6}\right)=-1, A_{5} / B_{1}=1, B_{3} /\left(B_{1} L^{2}\right)=-0.05$, $B_{5} / B_{1}=-10$ and $\tilde{F}=-1$ for (a) and $\tilde{F}=1$ for (b). $L$ is the period of the system.

mode consists of periodically alternating regions with nematic order at an angle of $\pi / 4$ and $-\pi / 4$ with the $x$-axis. These structures are reminiscent of chevrons in nematic liquid crystals, cf. Fig. 3(a) 22]. For $F<0$, the homogeneous state becomes unstable by either stationary instabilities or by Hopf bifurcations which lead to traveling wave solutions. Because of symmetry, these waves can propagate along the $x$-axis in both directions. Hopf bifurcations occur if $\left(D-B_{2}\right)^{2}+4 F<0$. For $F>0$ instabilities towards inhomogeneous states are always sta-

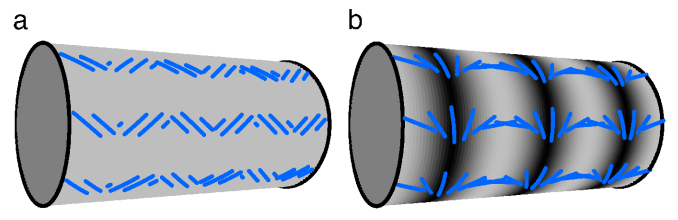

FIG. 3: (color online) Examples of stationary solutions to the dynamic equations with periodic boundary conditions. (a) Chevron pattern with homogeneous filament density for $\tilde{B}_{2}=$ $-1.25, \tilde{D}=0.75$, and $\tilde{F}=-1$. (b) Multiple ring pattern for $\tilde{B}_{2}=-1.5, \tilde{D}=1.5$, and $\tilde{F}=-1$.

tionary. The instabilities towards stationary states lead to periodic patterns of filament accumulation combined with nematic order, parallel or perpendicular to the $x$ axis, at the maxima of the filament density, see Fig. B(b). For decreasing $\tilde{B}_{2}$ the number of rings increases as the corresponding length scale decreases. In order to obtain the full phase diagrams displayed in Fig. 2, we solved the dynamic equations (3)-(5) numerically with periodic boundary conditions 23$]$.

Periodic boundary conditions are inappropriate to describe cells. An important boundary condition is the zero flux condition $\boldsymbol{j}_{0, L}=0$, which implies that the cortical material cannot leave the cell. Additional boundary conditions have to be specified to fully define the asymptotic solutions. It is observed that the end-faces of interphase plant cells are essentially free of microtubules. This is consistent with the absence of imposed order at the boundaries and we therefor set $\boldsymbol{q}_{0, L}=0$. In the following we use for simplicity the boundary conditions $\mathbf{j}=0$, $\mathbf{q}=0$ and $\partial_{x} c=\partial_{x} q_{x x}=\partial_{x} q_{x y}=0$ at both $x=0$ and $x=L$.

Figure 1(c) shows a steady state solution of Eqs. (3)(5) with zero flux boundary conditions. This solution corresponds to a stationary filament ring with filaments that are oriented in $y$-direction and localized in a ring-like pattern wound around the cylinder, strongly reminiscent of the PPB. The corresponding stress is anisotropic and for $\sigma_{y y}>\sigma_{x x}$ the ring solution is contractile. Additional asymptotic solutions are similar to the solutions shown in Fig. 3 with periodic boundary conditions. Furthermore, oscillatory solutions appear along a line of Hopf- bifurcations. An example of such a state with no flux boundary conditions is shown in Fig. [4 The oscillating solutions for no flux boundary conditions correspond to traveling waves in the case of periodic boundary conditions. Additional states can be generated via the boundaries by imposing other boundary conditions.

In conclusion we have presented a generic approach to the dynamics of a cortical layer of an active gel of cytoskeletal filaments near the cell surface. We have focussed on a situation which can describe the formation of filament rings such as the PPB in plant cells and the contractile ring in animal cells. Ringlike cytoskele- 


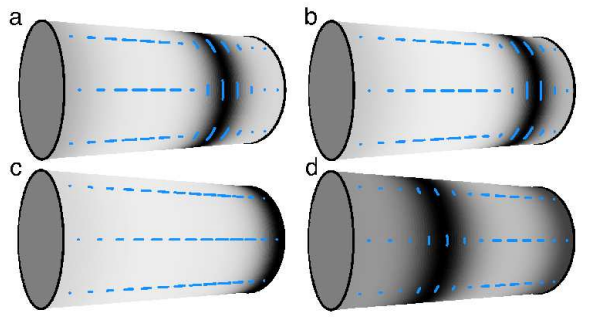

FIG. 4: (color online) Snapshots at different times of an oscillating filament ring for no-flux boundary conditions and $\tilde{B}_{2}=1, \tilde{D}=0.25$ and $\tilde{F}=1$. The pattern consists of a filament ring which forms near the center of the cylinder (a) and moves towards the right pole $(b, c)$, where the ring disappears. Simultaneously, a new ring is formed near the center (d) which subsequently moves to the opposite pole. The whole process is repeated periodically. The times correspond to phases $\phi=\omega t$ of the oscillation with $\phi=0$ (a), $\phi=(4 / 36) 2 \pi(\mathrm{b}), \phi=(7 / 36) 2 \pi(\mathrm{c})$ and $\phi=(15 / 36) 2 \pi(\mathrm{d})$.

tal patterns occur also in prokaryotes. An example is the Z-ring formed by the tubulin analog FtsZ [24]. We have analyzed the formation of patterns via dynamic instabilities resulting from active processes such as the action of motor proteins and the polymerization and depolymerization of filaments. Of particular interest is the spontaneous formation of filament rings which provides a possible mechanism for the formation of the PPB. Such ring structures can be contractile and could induce constriction of a deformable cylinder. Furthermore, we have found solutions corresponding to filament rings which move periodically towards both ends of the cylinder. Oscillating actin rings have been observed in lymphoblasts treated with nocodazole to remove the microtubules 25] and more recently in fragments of fibroblasts [26]. Qualitatively, they exhibit the same type of oscillatory pattern as described in Fig. 4]

Our description assumes that filaments are short as compared to the cylinder radius $R$. In the PPB, microtubules could have a length of several tens of $\mu \mathrm{m}$. In this case, the bending elasticity can become important and could lead to anisotropies in our description since azimuthal orientation would be energetically disfavored. In order to connect our macroscopic approach with molecular properties of filaments and associated proteins, more microscopic descriptions are valuable. For example, negative values of the parameter $\tilde{D}$ in the phase diagram of Fig. 2 can result from contractile stresses caused by the action of motor-aggregates [11, 15]. Similarly, the parameter $\tilde{B}_{2}$ characterizes a length scale associated with orientational order, which depends for example on filament lengths and packing density. Such relations between microscopic models and phenomenological descriptions could help to identify microscopic mechanisms, which underlie the formation of cortical rings in cells.

We thank Anne Mie Emons and Jan Vos for many stimulating discussions. The work of MCL, CT, BMM and MD is part of the research program of "Stichting Fundamenteel Onderzoek der Materie (FOM)" which is financially supported by "Nederlandse Organisatie voor Wetenschapelijke Onderzoek (NWO)".

* These authors contributed equally to this work.

$\dagger$ Current address:Institut Curie,Paris,France

¥ Current address:Institute of Theoretical Physics, Utrecht University, The Netherlands

[1] B. Alberts, A. Johnson, J. Lewis, M. Raff, K. Roberts, and P. Walter, Molecular Biology of the Cell, 4th Ed. Garland, 2002.

[2] G. O. Wasteneys, J. Cell. Sci. 115, 1345 (2002).

[3] C. L. Granger and J. Cyr, J. Cell. Sci. 114, 599 (2001).

[4] A. Yoneda, M. Akatsuka, H. Hoshino, F. Kumagai, and S. Hasezawa, Plant Cell Physiol. 46, 531 (2005).

[5] F. J. Nedelec, T. Surrey, A. C. Maggs, and S. Leibler, Nature 389, 305 (1997).

[6] T. Surrey, F. Nédélec, S. Leibler, and E. Karsenti, Science 292,1167 (2001).

[7] F. Nedelec, J. Cell Biol. 158,1005 (2002).

[8] K. Kruse and F. Jülicher, Phys. Rev. Lett. 85, 1778 (2000).

[9] B. Bassetti, M.C. Lagomarsino, and P. Jona, Eur. Phys. Jour. B 15, 483 (2000).

[10] K. Kruse and F. Jülicher, Phys. Rev. E 67, 051913 (2003).

[11] T. B. Liverpool and M. C. Marchetti, Phys. Rev. Lett. 90, 138102 (2003), Europhys. Lett. 69, 846 (2005).

[12] H. Y. Lee and M. Kardar, Phys. Rev. E 64, 056113 (2001).

[13] J. Kim, Y. Park, B. Kahng, and H. Y. Lee, J. Korean Phys. Soc. 42, 162 (2003).

[14] S. Sankararaman, G. I. Menon, and P. B. Sunil Kumar, Phys. Rev. E 70, 031905 (2004).

[15] K. Kruse, A. Zumdieck, and F. Jülicher, Europhys. Lett. 64, 716 (2003).

[16] K. Kruse, J.F. Joanny, F. Jülicher, J. Prost, and K. Sekimoto, Phys. Rev. Lett. 92, 078101 (2004), Eur. Phys. J. E 16, 5 (2005).

[17] F. Ziebert and W. Zimmermann, Phys. Rev. E 70, 022902 (2004).

[18] J. Chan, G. M. Calder, J. H. Doonan, and C. W. Lloyd, Nat. Cell Biol. 5, 967 (2003).

[19] G. W. Tian, D. Smith, S. Gluck, and T. I. Baskin, Cell Motil. Cytoskeleton 57, 26 (2004).

[20] P. Dhonukshe and T. W. Gadella, Jr., Plant Cell 15, 597 (2003).

[21] J. W. Vos, M. Dogterom, and A. M. C. Emons, Cell Motil. Cytoskeleton 57, 246 (2004).

[22] P.G. De Gennes and J. Prost, The physics of liquid crystals, Clarendon Press (1993).

[23] We used a forward Euler scheme to numerically integrate Eqs. (1)-(5). Space was discretized in steps of $\Delta x=0.01 L$. The time step was $\Delta t=10^{-7} / B_{1}$.

[24] J. Lutkenhaus, Curr. Opin. Microbiol. 5, 548 (2002).

[25] M. Bornens, M. Paintrand, and C. Celati, J. Cell Biol. 109, 1071 (1989).

[26] E. Paluch, M. Piel, J Prost, M Bornens, C Sykes, Bio- 
phys. J. 89, 724 (2005).

[27] C. Tanase and B. Mulder, unpublished. 